Article

\title{
Multiple Attribute Decision-Making Method Using Similarity Measures of Neutrosophic Cubic Sets
}

\author{
Angyan Tu ${ }^{1,2}$ (D), Jun $\mathrm{Ye}^{2}$ (D) and Bing Wang ${ }^{1, *}$ \\ 1 School of Mechatronic Engineering and Automation, Shanghai University, 149 Yanchang Road, \\ Shanghai 200072, China; lucytu@shu.edu.cn \\ 2 Department of Electrical and Information Engineering, Shaoxing University, 508 Huancheng West Road, \\ Shaoxing 312000, China; yejun@usx.edu.cn \\ * Correspondence: susanbwang@shu.edu.cn
}

Received: 18 May 2018; Accepted: 11 June 2018; Published: 12 June 2018

\begin{abstract}
In inconsistent and indeterminate settings, as a usual tool, the neutrosophic cubic set (NCS) containing single-valued neutrosophic numbers and interval neutrosophic numbers can be applied in decision-making to present its partial indeterminate and partial determinate information. However, a few researchers have studied neutrosophic cubic decision-making problems, where the similarity measure of NCSs is one of the useful measure methods. For this work, we propose the Dice, cotangent, and Jaccard measures between NCSs, and indicate their properties. Then, under an NCS environment, the similarity measures-based decision-making method of multiple attributes is developed. In the decision-making process, all the alternatives are ranked by the similarity measure of each alternative and the ideal solution to obtain the best one. Finally, two practical examples are applied to indicate the feasibility and effectiveness of the developed method.
\end{abstract}

Keywords: similarity measures; neutrosophic cubic set; decision-making

\section{Introduction}

The classic fuzzy set [1] is expressed by its membership degree in the unit interval [0,1]. But in many complicated cases of the real world, the data often are vague and uncertain, and are difficult to express as classic fuzzy sets. Thus, the neutrosophic set (NS) concept was presented by Smarandache [2], which is an extension of the fuzzy set and (interval-valued) intuitionistic fuzzy sets. He defined the indeterminacy, falsity, and truth degrees of NS in the nonstandard interval ]$^{-} 0,1^{+}$[and standard interval $[0,1]$. However, the nonstandard interval is difficult to apply in real situations, so a simplified neutrosophic set (SNS), including single-valued and interval neutrosophic sets, was presented by Ye [3], which is depicted by the truth, indeterminacy, and falsity degrees in the interval [0,1], to conveniently apply it in science and engineering fields, such as decision-making [4-8], medical diagnoses [9,10], image processing [11,12], and clustering analyses [13]. Meanwhile, different measures were constantly proposed, such as similarity measures, cross entropy measures, correlation coefficients, and various aggregation operators for multiple attribute decision-making (MADM) problems [14-21]. Then, various simplified neutrosophic decision-making methods were presented, such as the technique for order preference by similarity to an ideal solution (TOPSIS) method [22], the projection and bidirectional projection measures [23], and the VIKOR method [24].

In recent years, (fuzzy) cubic sets (CSs) presented by Jun et al. [25] have received much attention due to the vague properties of human hesitant judgments. Since CS implies its partial certain and partial uncertain information, it is depicted by the hybrid form composed of an exact value and an interval value. Hence, CSs are very well suited for the representation of its partial indeterminate and partial determinate information in fuzzy environments. But many scientific problems in the 
real world are very complex. To handle more complicated problems with incomplete, inconsistent, and indeterminate information, Jun et al. [26] and Ali et al. [27] have introduced neutrosophic cubic sets (NCSs) which contain both single-valued neutrosophic information and interval neutrosophic information, as introduced in References [2,28,29]. Lu and Ye [30] used cosine measures for NCSs for the first time to handle decision-making problems in an NCS setting. Banerjee et al. [31] presented MADM problems regarding grey relational analysis in an NCS setting. Pramanik et al. [32] introduced a multiple attribute group decision-making method regarding the distance-based similarity measure of NCSs. Ye [33] put forward the operational laws and weighted aggregation operators of NCSs and their MADM method in an NCS setting. Then, Shi and Ye [34] further proposed the Dombi aggregation operators of NCSs and their MADM method. However, few researchers have studied neutrosophic cubic MADM problems, where the similarity measure of NCSs is one of the useful measure methods. On the other hand, Ye proposed the cosine, Dice, and Jaccard measures of single-valued and interval neutrosophic sets [35], the generalized Dice measure of SNSs [36], and the single-valued neutrosophic cotangent measures [37]. Since NCS is combined with an interval neutrosophic set (INS) and a single-valued neutrosophic set (SVNS), we can extend them to NCSs. Motivated by the similarity measures of INSs and SVNSs in the literature [35,37], we propose the Dice, cotangent, and Jaccard measures between NCSs to enrich the existing similarity measures of NCSs. Then, a MADM method is developed based on the proposed similarity measures in an NCS setting. Their difference is that the similarity measures in the literature [30] only use three cosine measures for MADM problems, but this work proposes the Dice, cotangent, and Jaccard measures for MADM problems in an NCS setting. By comparison with existing decision-making methods [30], the decision results show that our similarity measures have better decision-making robustness and discrimination than existing cosine measures [30].

The contents of this paper are organized as follows: Section 2 introduces basic definitions of CSs and NCSs. The similarity measures of NCSs and their properties are presented in Section 3. A MADM method is developed by using the three measures of the Dice, cotangent, and Jaccard measures in Section 4. In Section 5, a practical example is given in an NCS setting to present the applications and the effectiveness of the developed method. Finally, Section 6 indicates conclusions and future work.

\section{Basic Definitions of CSs and NCSs}

Based on the combination of both a fuzzy value and an interval-valued fuzzy number (IVFN), a CS was defined by Jun et al. [25].

The CS $Z$ in a universe of discourse $Y$ is defined by the following form [25]:

$$
Z=\{y, T(y), \mu(y) \mid y \in Y\},
$$

where $\mu(y)$ is a fuzzy value and $T(y)=\left[T^{-}(y), T^{+}(y)\right]$ is an IVFN for $y \in Y$. Then, we define

(i) $Z=\{y, T(y), \mu(y) \mid y \in Y\}$ as an internal CS if $T^{-}(y) \leq \mu(y) \leq T^{+}(y)$ for $y \in Y$;

(ii) $Z=\{y, T(y), \mu(y) \mid y \in Y\}$ as an external CS if $\mu(y) \notin\left[T^{-}(y), T^{+}(y)\right]$ for $y \in Y$.

When combining a single-valued neutrosophic number (SVNN) with an interval neutrosophic number (INN), CS was extended to NCS by Jun et al. [26] and Ali et al. [27], which is constructed as an NCS $Z$ in $Y$ by the following form [26,27]:

$$
R=\{y,<T(y), U(y), F(y)>t(y), u(y), f(y)>y \in Y\},
$$

where $<T(y), U(y), F(y)>$ is an INN for the truth-interval $T(y)=\left[T^{-}(y), T^{+}(y)\right] \subseteq[0,1]$, the falsityinterval $F(y)=\left[F^{-}(y), F^{+}(y)\right] \subseteq[0,1]$, the indeterminacy-interval $U(y)=\left[U^{-}(y), U^{+}(y)\right] \subseteq[0,1]$, $y \in Y$ and $<t(y), \mathrm{u}(y), \mathrm{f}(y)>$ is an SVNN for the truth, falsity, and indeterminacy degrees $t(y), f(y), u(y) \in[0,1]$ and $y \in Y$.

An NCS $R=\{y,<T(y), U(y), F(y)>t(y), u(y), f(y)>y \in Y\}$ is called [26,27]: 
(i) An internal NCS $R=\{y,<T(y), U(y), F(y)>t(y), u(y), f(y)>y \in Y\}$ if $T^{-}(y) \leq t(y) \leq$ $T^{+}(y), U^{-}(y) \leq u(y) \leq U^{+}(y)$, and $F^{-}(y) \leq f(y) \leq F^{+}(y)$ for $y \in Y$;

(ii) An external NCS $R=\{y,<T(y), U(y), F(y)>t(y), u(y), f(y)>y \in Y\}$ if $t(y) \notin$ $\left[T^{-}(y), T^{+}(y)\right], u(y) \notin\left[U^{-}(y), U^{+}(y)\right]$, and $f(y) \notin\left[F^{-}(y), F^{+}(y)\right]$ for $y \in Y$.

For the simplified expression, a basic element $(y,<T(y), U(y), F(y)>t(y), u(y), f(y)>$ in an NCS $R$ is denoted as $r=(\langle T, U, F\rangle t, u, f\rangle$, which is called a neutrosophic cubic number $(\mathrm{NCN})$, where $T, U, F \subseteq[0,1]$ and $t, u, f \in[0,1]$, satisfying $0 \leq T^{+}(y)+U^{+}(y)+F^{+}(y) \leq 3$ and $0 \leq t+u+f \leq 3$.

Let $r_{1}=\left(<T_{1}, U_{1}, F_{1}>t_{1}, u_{1}, f_{1}>\right.$ and $r_{2}=\left(<T_{2}, U_{2}, F_{2}>t_{2}, u_{2}, f_{2}>\right.$ be two NCNs. We can indicate the following relations [26,27]:

(1) $\left.r_{1}^{\mathrm{c}}=\left(<F_{1}^{-}, F_{1}^{+}\right],\left[1-U_{1}^{+}, 1-U_{1}^{-}\right],\left[T_{1}^{-}, T_{1}^{+}\right]>f_{1}, 1-u_{1}, t_{1}\right)$ (the complement of $\left.r_{1}\right)$;

(2) $r_{1} \subseteq r_{2}$ if and only if $T_{1} \subseteq T_{2}, U_{1} \supseteq U_{2}, F_{1} \supseteq F_{2}, t_{1} \leq t_{2}, u_{1} \geq u_{2}$, and $f_{1} \geq f_{2}$ (P-order);

(3) $\quad r_{1}=r_{2}$ if and only if $r_{1} \subseteq r_{2}$ and $r_{2} \subseteq r_{1}$, i.e., $<T_{1}, U_{1}, F_{1}>T_{2}, U_{2}, F_{2}>$ and $<t_{1}, u_{1}, f_{1}>t_{2}, u_{2}, f_{2}>$.

\section{Similarity Measures of NCSs}

Based on the Dice and Jaccard measures of SVNSs and INSs (SNSs) [35], and the single-valued neutrosophic cotangent measures [37] proposed by Ye, we can extend them to NCSs to present the Dice, Jaccard, and cotangent measures between NCSs in this section.

Definition 1. Let two NCSs beR $=\{r 1, r 2, r 3, \cdots, r n\}$ and $H=\{h 1, h 2, h 3, \cdots, h n\}$ in the universe of discourse $Y=\{y 1, y 2, y 3, \cdots, y n\}$, where $r_{i}=\left(<T_{r i}, U_{r i}, F_{r i}>t_{r i}, u_{r i}, f_{r i}>\right.$ and $h_{i}=\left(<T_{h i}, U_{h i}, F_{h i}>\right.$ $t_{h i}, u_{h i}, f_{h i}>$ are two NCNs for $i=1,2, \ldots, n$. Thus, the similarity measures of the NCSs $R$ and $H$ are presented as follows:

(1) Dice Measure between the NCSs R and H

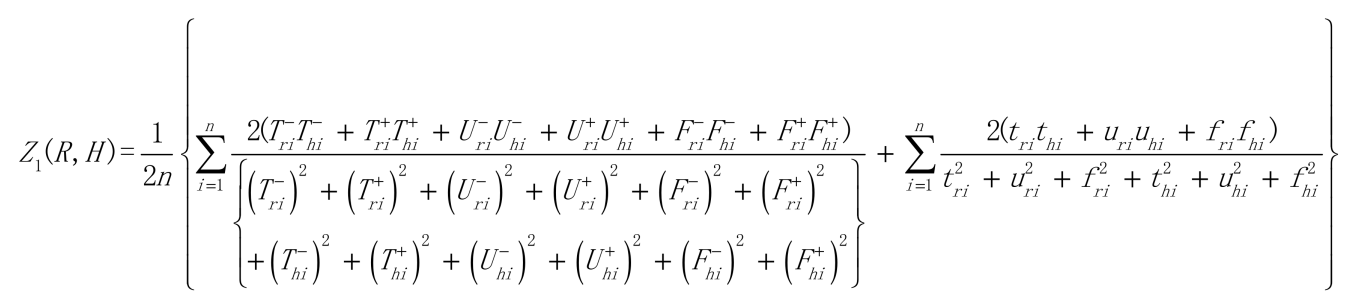

(2) Cotangent Measure between the NCSs R and H

$$
Z_{2}(R, H)=\frac{1}{2 n} \sum_{i=1}^{n}\left\{\begin{array}{l}
\cot \left[\frac{\pi}{4}+\frac{\pi}{24}\left(\left|T_{r i}^{-}-T_{h i}^{-}\right|+\left|T_{r i}^{+}-T_{h i}^{+}\right|+\left|U_{r i}^{-}-U_{h i}^{-}\right|+\left|U_{r i}^{+}-U_{h i}^{+}\right|+\left|F_{r i}^{+}-F_{h i}^{-}\right|+\left|F_{r i}^{+}-F_{h i}^{+}\right|\right)\right. \\
+\cot \left[\frac{\pi}{4}+\frac{\pi}{12}\left(\left|t_{r i}-t_{h i}\right|+\left|u_{r i}-u_{h i}\right|+\mid f_{r i}-f_{h i}\right)\right]
\end{array}\right\}
$$


(3) Jaccard Measure between the NCSs $R$ and $H$

$$
\left.Z_{3}(R, H)=\frac{1}{2 n}\left\{\begin{array}{l}
\sum_{i=1}^{n} \frac{T_{r i}^{-} T_{h i}^{-}+T_{r i}^{+} T_{h i}^{+}+U_{r i}^{-} U_{h i}^{-}+U_{r i}^{+} U_{h i}^{+}+F_{r i}^{-} F_{h i}^{-}+F_{r i}^{+} F_{h i}^{+}}{\left(T_{r i}^{-}\right)^{2}+\left(T_{r i}^{+}\right)^{2}+\left(U_{r i}^{-}\right)^{2}+\left(U_{r i}^{+}\right)^{2}+\left(F_{r i}^{-}\right)^{2}+\left(F_{r i}^{+}\right)^{2}} \\
+\left(T_{h i}^{-}\right)^{2}+\left(T_{h i}^{+}\right)^{2}+\left(U_{h i}^{-}\right)^{2}+\left(U_{h i}^{+}\right)^{2}+\left(F_{h i}^{-}\right)^{2}+\left(F_{h i}^{+}\right)^{2} \\
-T_{r i}^{-} T_{h i}^{-}-T_{r i}^{+} T_{h i}^{+}-U_{r i}^{-} U_{h i}^{-}-U_{r i}^{+} U_{h i}^{+}-F_{r i}^{-} F_{h i}^{-}-F_{r i}^{+} F_{h i}^{+}
\end{array}\right\} \sum_{i=1}^{n} \frac{t_{r i} t_{h i}+u_{r i} u_{h i}+f_{r i} f_{h i}}{\left\{\begin{array}{l}
\left.t_{r i}^{2}+u_{r i}^{2}+f_{r i}^{2}+t_{h i}^{2}+u_{h i}^{2}+f_{h i}^{2}\right) \\
-t_{r i} t_{h i}-u_{r i} u_{h i}-f_{r i} f_{h i}
\end{array}\right\}}\right\}
$$

Theorem 1. The three measures $Z_{m}(R, H)(m=1,2,3)$ satisfy the three properties (I)-(III):

(I) $0 \leq Z_{m}(R, H) \leq 1$;

(II) $Z_{m}(R, H)=Z_{m}(H, R)$;

(III) $Z_{m}(R, H)=1$ if $R=H$, i.e., $<T_{r i}, U_{r i}, F_{r i}>=<T_{h i}, U_{h i}, F_{h i}>$ and $<t_{r i}, u_{r i}, f_{r i}><t_{h i}, u_{h i}, f_{h i}>$.

\section{Proof.}

Firstly, we prove the properties (I)-(III) of $\left.Z_{1}(R, H)\right)$.

(I) The inequality $Z_{1}(R, H) \geq 0$ is obvious. Then, we only prove $Z_{1}(R, H) \leq 1$.

Based on the basic inequality $2 x_{i} y_{i} \leq x_{i}^{2}+y_{i}^{2}$ for $i=1,2, \ldots, n$, where $\left(x_{1}, x_{2}, x_{3}, \ldots, x_{n}\right) \in R^{n}$ and $\left(y_{1}, y_{2}, y_{3}, \ldots, y_{n}\right) \in R^{n}$, it is extended to the NCNs, and then the following inequality is obtained:

$$
2\left(T_{r i}^{-} T_{h i}^{-}\right) \leq\left(T_{r i}^{-}\right)^{2}+\left(T_{h i}^{-}\right)^{2}
$$

When $T_{r i}^{-}$and $T_{h i}^{-}$are not equal to zero, we obtain the following inequality:

$$
\frac{2\left(T_{r i}^{-} T_{h i}^{-}\right)}{\left(T_{r i}^{-}\right)^{2}+\left(T_{h i}^{-}\right)^{2}} \leq 1
$$

Similarly, we have these inequalities $2\left(T_{r i}^{+} T_{h i}^{+}\right) \leq\left(T_{r i}^{+}\right)^{2}+\left(T_{h i}^{+}\right)^{2}, 2\left(U_{r i}^{-} U_{h i}^{-}\right) \leq\left(U_{r i}^{-}\right)^{2}+\left(U_{h i}^{-}\right)^{2}$, $2\left(U_{r i}^{+} U_{h i}^{+}\right) \leq\left(U_{r i}^{+}\right)^{2}+\left(U_{h i}^{+}\right)^{2}, 2\left(F_{r i}^{-} F_{h i}^{-}\right) \leq\left(F_{r i}^{-}\right)^{2}+\left(F_{h i}^{-}\right)^{2}$, and $2\left(F_{r i}^{+} F_{h i}^{+}\right) \leq\left(F_{r i}^{+}\right)^{2}+\left(F_{h i}^{+}\right)^{2}$.

Then, we get the following sum of the six inequalities with both sides.

$$
\begin{gathered}
2\left(T_{r i}^{-} T_{h i}^{-}\right)+2\left(T_{r i}^{+} T_{h i}^{+}\right)+2\left(U_{r i}^{-} U_{h i}^{-}\right)+2\left(U_{r i}^{+} U_{h i}^{+}\right)+2\left(F_{r i}^{-} F_{h i}^{-}\right)+2\left(F_{r i}^{+} F_{h i}^{+}\right) \leq \\
\left(T_{r i}^{-}\right)^{2}+\left(T_{h i}^{-}\right)^{2}+\left(T_{r i}^{+}\right)^{2}+\left(T_{h i}^{+}\right)^{2}+\left(U_{r i}^{-}\right)^{2}+\left(U_{h i}^{-}\right)^{2}+\left(U_{r i}^{+}\right)^{2}+\left(U_{h i}^{+}\right)^{2}+\left(F_{r i}^{-}\right)^{2}+\left(F_{h i}^{-}\right)^{2}+\left(F_{r i}^{+}\right)^{2}+\left(F_{h i}^{+}\right)^{2}
\end{gathered}
$$

Thus, we have the following result:

$$
\frac{2\left(T_{r i}^{-} T_{h i}^{-}\right)+2\left(T_{r i}^{+} T_{h i}^{+}\right)+2\left(U_{r i}^{-} U_{h i}^{-}\right)+2\left(U_{r i}^{+} U_{h i}^{+}\right)+2\left(F_{r i}^{-} F_{h i}^{-}\right)+2\left(F_{r i}^{+} F_{h i}^{+}\right)}{\left\{\begin{array}{c}
\left(T_{r i}^{-}\right)^{2}+\left(T_{h i}^{-}\right)^{2}+\left(T_{r i}^{+}\right)^{2}+\left(T_{h i}^{+}\right)^{2}+\left(U_{r i}^{-}\right)^{2}+\left(U_{h i}^{-}\right)^{2} \\
+\left(U_{r i}^{+}\right)^{2}+\left(U_{h i}^{+}\right)^{2}+\left(F_{r i}^{-}\right)^{2}+\left(F_{h i}^{-}\right)^{2}+\left(F_{r i}^{+}\right)^{2}+\left(F_{h i}^{+}\right)^{2}
\end{array}\right\}} \leq 1 .
$$

So, we can further get the result:

$$
\frac{1}{n} \sum_{i=1}^{n} \frac{2\left(T_{r i}^{-} T_{h i}^{-}\right)+2\left(T_{r i}^{+} T_{h i}^{+}\right)+2\left(U_{r i}^{-} U_{h i}^{-}\right)+2\left(U_{r i}^{+} U_{h i}^{+}\right)+2\left(F_{r i}^{-} F_{h i}^{-}\right)+2\left(F_{r i}^{+} F_{h i}^{+}\right)}{\left\{\begin{array}{l}
\left(T_{r i}^{-}\right)^{2}+\left(T_{h i}^{-}\right)^{2}+\left(T_{r i}^{+}\right)^{2}+\left(T_{h i}^{+}\right)^{2}+\left(U_{r i}^{-}\right)^{2}+\left(U_{h i}^{-}\right)^{2} \\
+\left(U_{r i}^{+}\right)^{2}+\left(U_{h i}^{+}\right)^{2}+\left(F_{r i}^{-}\right)^{2}+\left(F_{h i}^{-}\right)^{2}+\left(F_{r i}^{+}\right)^{2}+\left(F_{h i}^{+}\right)^{2}
\end{array}\right\}} \leq 1 .
$$


Similarly, we have the following inequalities:

$$
\frac{1}{n} \sum_{i=1}^{n} \frac{2\left(t_{r i} t_{h i}+u_{r i} u_{h i}+f_{r i} f_{h i}\right)}{t_{r i}^{2}+u_{r i}^{2}+f_{r i}^{2}+t_{h i}^{2}+u_{h i}^{2}+f_{h i}^{2}} \leq 1
$$

Thus, we have $Z_{1}(R, H) \leq 1$, and then $0 \leq Z_{1}(R, H) \leq 1$ holds.

(II) The equality is obvious.

(III) When $R=H$, we have $\left\langle T_{r i}, U_{r i}, F_{r i}\right\rangle=\left\langle T_{h i}, U_{h i}, F_{h i}\right\rangle$ and $\left\langle t_{r i}, u_{r i}, f_{r i}><t_{h i}, u_{h i}, f_{h i}\right\rangle$. Thus $T_{r i}=T_{h i}, U_{r i}=U_{h i}, F_{r i}=F_{h i}, t_{r i}=t_{h i}, u_{r i}=u_{h i}$, and $f_{r i}=f_{h i}$ for $i=1,2, \ldots, n$. Hence $Z_{1}(R, H)=1$ holds.

Secondly, the properties (I)-(III) of $Z_{2}(R, H)$ can be proved as follows:

(I) The inequality $0 \leq\left|T_{r i}^{-}-T_{h i}^{-}\right| \leq 1$ is obvious. Similarly, we obtain other inequalities $0 \leq\left|T_{r i}^{+}-T_{h i}^{+}\right| \leq 1, \quad 0 \leq\left|U_{r i}^{-}-U_{h i}^{-}\right| \leq 1, \quad 0 \leq\left|U_{r i}^{+}-U_{h i}^{+}\right| \leq 1, \quad 0 \leq\left|F_{r i}^{-}-F_{h i}^{-}\right| \leq 1$, and $0 \leq\left|F_{r i}^{+}-F_{h i}^{+}\right| \leq 1$.

Based on these inequalities, we get the inequality:

$0 \leq\left|T_{r i}^{-}-T_{h i}^{-}\right|+\left|T_{r i}^{+}-T_{h i}^{+}\right|+\left|U_{r i}^{-}-U_{h i}^{-}\right|+\left|U_{r i}^{+}-U_{h i}^{+}\right|+\left|F_{r i}^{-}-F_{h i}^{-}\right|+\left|F_{r i}^{+}-F_{h i}^{+}\right| \leq 6$, and then obtain the inequality:

$0 \leq \frac{1}{24}\left(\left|T_{r i}^{-}-T_{h i}^{-}\right|+\left|T_{r i}^{+}-T_{h i}^{+}\right|+\left|U_{r i}^{-}-U_{h i}^{-}\right|+\left|U_{r i}^{+}-U_{h i}^{+}\right|+\left|F_{r i}^{-}-F_{h i}^{-}\right|+\left|F_{r i}^{+}-F_{h i}^{+}\right|\right) \leq \frac{1}{4}$

and the following inequality:

$0 \leq \frac{\pi}{24}\left(\left|T_{r i}^{-}-T_{h i}^{-}\right|+\left|T_{r i}^{+}-T_{h i}^{+}\right|+\left|U_{r i}^{-}-U_{h i}^{-}\right|+\left|U_{r i}^{+}-U_{h i}^{+}\right|+\left|F_{r i}^{-}-F_{h i}^{-}\right|+\left|F_{r i}^{+}-F_{h i}^{+}\right|\right) \leq \frac{\pi}{4}$.

Hence, the result is obtained as follows:

$\cot \left(\frac{\pi}{2}\right) \leq \cot \left[\frac{\pi}{4}+\frac{\pi}{24}\left(\left|T_{r i}^{-}-T_{h i}^{-}\right|+\left|T_{r i}^{+}-T_{h i}^{+}\right|+\left|U_{r i}^{-}-U_{h i}^{-}\right|+\left|U_{r i}^{+}-U_{h i}^{+}\right|+\left|F_{r i}^{-}-F_{h i}^{-}\right|+\left|F_{r i}^{+}-F_{h i}^{+}\right|\right)\right] \leq \cot \left(\frac{\pi}{4}\right)$.

Simplifying the above inequality, we get the simplified inequality:

$0 \leq \cot \left[\frac{\pi}{4}+\frac{\pi}{24}\left(\left|T_{r i}^{-}-T_{h i}^{-}\right|+\left|T_{r i}^{+}-T_{h i}^{+}\right|+\left|U_{r i}^{-}-U_{h i}^{-}\right|+\left|U_{r i}^{+}-U_{h i}^{+}\right|+\left|F_{r i}^{-}-F_{h i}^{-}\right|+\left|F_{r i}^{+}-F_{h i}^{+}\right|\right)\right] \leq 1$.

Let us prove the other inequality $0 \leq \cot \left[\frac{\pi}{4}+\frac{\pi}{12}\left(\left|t_{r i}-t_{h i}\right|+\left|u_{r i}-u_{h i}\right|+\left|f_{r i}-f_{h i}\right|\right)\right] \leq 1$. Because there are the inequalities $0 \leq\left|t_{r i}-t_{h i}\right| \leq 1,0 \leq\left|u_{r i}-u_{h i}\right| \leq 1$, and $0 \leq\left|f_{r i}-f_{h i}\right| \leq 1$, we get the inequality $0 \leq\left|t_{r i}-t_{h i}\right|+\left|u_{r i}-u_{h i}\right|+\left|f_{r i}-f_{h i}\right| \leq 1$ and $0 \leq \frac{\pi}{12}\left(\left|t_{r i}-t_{h i}\right|+\left|u_{r i}-u_{h i}\right|+\left|f_{r i}-f_{h i}\right|\right) \leq$ $\frac{3 \pi}{12}$, and then $\cot \left(\frac{\pi}{2}\right) \leq \cot \left[\frac{\pi}{4}+\frac{\pi}{12}\left(\left|t_{r i}-t_{h i}\right|+\left|u_{r i}-u_{h i}\right|+\left|f_{r i}-f_{h i}\right|\right)\right] \leq \cot \left(\frac{\pi}{4}\right)$. Thus, the other form is $0 \leq \cot \left[\frac{\pi}{4}+\frac{\pi}{12}\left(\left|t_{r i}-t_{h i}\right|+\left|u_{r i}-u_{h i}\right|+\left|f_{r i}-f_{h i}\right|\right)\right] \leq 1$. Hence $0 \leq Z_{2}(R, H) \leq 1$ holds.

Thirdly, the properties (I)-(III) of $Z_{3}(R, H)$ can be proved below.

Based on the inequality $x y \leq x^{2}+y^{2}-x y$, we get such an inequality $T_{r i}^{-} T_{h i}^{-} \leq\left(T_{r i}^{-}\right)^{2}+\left(T_{h i}^{-}\right)^{2}-T_{r i}^{-} T_{h i}^{-}$. When $T_{r i}^{-}$and $T_{h i}^{-}$are not equal to zero, we obtain the inequality:

$$
\frac{T_{r i}^{-} T_{h i}^{-}}{\left(T_{r i}^{-}\right)^{2}+\left(T_{h i}^{-}\right)^{2}-T_{r i}^{-} T_{h i}^{-}} \leq 1 .
$$

Thus, we can get the following inequality:

$$
\frac{T_{r i}^{-} T_{h i}^{-}+T_{r i}^{+} T_{h i}^{+}+U_{r i}^{-} U_{h i}^{-}+U_{r i}^{+} U_{h i}^{+}+F_{r i}^{-} F_{h i}^{-}+F_{r i}^{+} F_{h i}^{+}}{\left\{\begin{array}{c}
\left(T_{r i}^{-}\right)^{2}+\left(T_{r i}^{+}\right)^{2}+\left(U_{r i}^{-}\right)^{2}+\left(U_{r i}^{+}\right)^{2}+\left(F_{r i}^{-}\right)^{2}+\left(F_{r i}^{+}\right)^{2} \\
+\left(T_{h i}^{-}\right)^{2}+\left(T_{h i}^{+}\right)^{2}+\left(U_{h i}^{-}\right)^{2}+\left(U_{h i}^{+}\right)^{2}+\left(F_{h i}^{-}\right)^{2}+\left(F_{h i}^{+}\right)^{2} \\
-T_{r i}^{-} T_{h i}^{-}-T_{r i}^{+} T_{h i}^{+}-U_{r i}^{-} U_{h i}^{-}-U_{r i}^{+} U_{h i}^{+}-F_{r i}^{-} F_{h i}^{-}-F_{r i}^{+} F_{h i}^{+}
\end{array}\right\}} \leq 1 .
$$


Similarly, because the inequality $\frac{t_{r i} t_{h i}}{t_{r i}^{2}+t_{h i}^{2}-t_{r i} t_{h i}} \leq 1$ holds, the inequality $\frac{t_{r i} h_{h i}+u_{r i} u_{h i}+f_{r i} f_{h i}}{t_{r i}^{2}+u_{r i}^{2}+f_{r i}^{2}+t_{h i}^{2}+u_{h i}^{2}+f_{h i}^{2}-t_{r i} t_{h i}-u_{r i} u_{h i}-f_{r i} f_{h i}} \leq 1$ also holds. Hence, there is the following inequality:

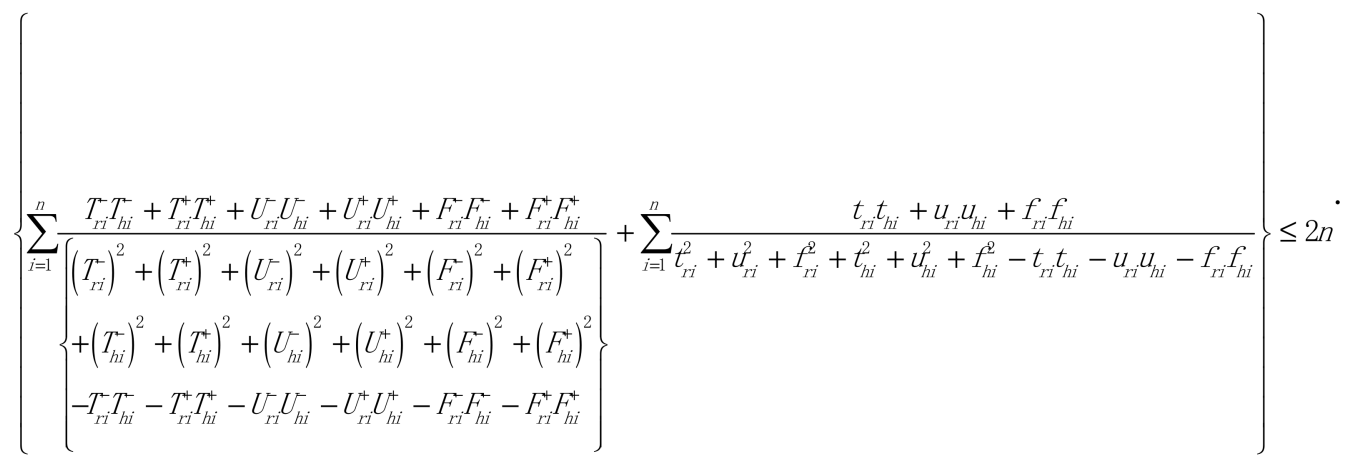

Thus, we have $Z_{3}(R, H) \leq 1$. Then, $0 \leq Z_{3}(R, H) \leq 1$ holds.

If we consider $\theta=\left\{\theta_{1}, \theta_{2}, \cdots, \theta_{n}\right\}$ as the weights of the elements $r_{i}$ and $h_{i}$ with $\theta_{i} \in[0,1]$ and $\sum_{i=1}^{n} \theta_{i}=1$, the corresponding three measures $Z_{\theta m}(R, H)(m=1,2,3)$ are given as follows:

$$
\left.\begin{array}{c}
Z_{\theta 1}(R, H)=\frac{1}{2}\left\{\begin{array}{c}
\sum_{i=1}^{n} \theta_{i} \frac{2\left(T_{r i}^{-} T_{h i}^{-}+T_{r i}^{+} T_{h i}^{+}+U_{r i}^{-} U_{h i}^{-}+U_{r i}^{+} U_{h i}^{+}+F_{r i}^{-} F_{h i}^{-}+F_{r i}^{+} F_{h i}^{+}\right)}{\left(T_{r i}^{-}\right)^{2}+\left(T_{r i}^{+}\right)^{2}+\left(U_{r i}^{-}\right)^{2}+\left(U_{r i}^{+}\right)^{2}+\left(F_{r i}^{-}\right)^{2}+\left(F_{r i}^{+}\right)^{2}} \\
+\left(T_{h i}^{-}\right)^{2}+\left(T_{h i}^{+}\right)^{2}+\left(U_{h i}^{-}\right)^{2}+\left(U_{h i}^{+}\right)^{2}+\left(F_{h i}^{-}\right)^{2}+\left(F_{h i}^{+}\right)^{2}
\end{array}\right\}+\sum_{i=1}^{n} \theta_{i} \frac{2\left(t_{r i} t_{h i}+u_{r i} u_{h i}+f_{r i} f_{h i}\right)}{t_{r i}^{2}+u_{r i}^{2}+f_{r i}^{2}+t_{h i}^{2}+u_{h i}^{2}+f_{h i}^{2}} \\
Z_{\theta 2}(R, H)=\frac{1}{2} \sum_{i=1}^{n} \theta_{i}\left\{\begin{array}{l}
\cot \left[\frac{\pi}{4}+\frac{\pi}{24}\left(\left|T_{r i}^{-}-T_{h i}^{-}\right|+\left|T_{r i}^{+}-T_{h i}^{+}\right|+\left|U_{r i}^{-}-U_{h i}^{-}\right|+\left|U_{r i}^{+}-U_{h i}^{+}\right|+\left|F_{r i}^{-}-F_{h i}^{-}\right|+\left|F_{r i}^{+}-F_{h i}^{+}\right|\right)\right. \\
+\cot \left[\frac{\pi}{4}+\frac{\pi}{12}\left(\left|t_{r i}-t_{h i}\right|+\left|u_{r i}-u_{h i}\right|+\left|f_{r i}-f_{h i}\right|\right)\right.
\end{array}\right]
\end{array}\right\}
$$

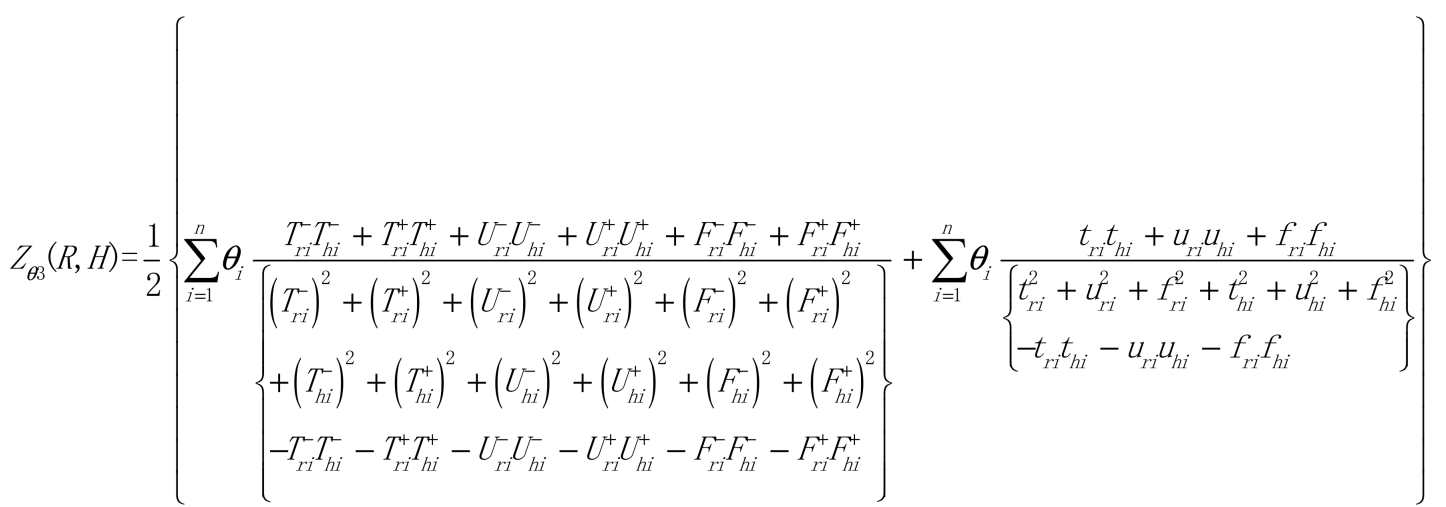

Obviously, the three measures $Z_{\theta m}(R, H)(m=1,2,3)$ also conform to the following properties (I)-(III):

(I) $0 \leq Z_{\theta m}(R, H) \leq 1$;

(II) $Z_{\theta m}(R, H)=Z_{\theta m}(H, R)$;

(III) $Z_{\theta m}(R, H)=1$ if $R=H$, i.e., $\left\langle t_{r i}, u_{r i}, f_{r i}\right\rangle=\left\langle t_{h i}, u_{h i}, f_{h i}\right\rangle$ and $\left\langle T_{r i}, U_{r i}, F_{r i}\right\rangle=\left\langle T_{h i}, U_{h i}, F_{h i}\right\rangle$.

The proofs of the three properties are similar, so we omitted them here. 


\section{MADM Method Using the Proposed Measures of NCSs}

The proposed weighted measures of NCSs are applied in MADM problems with NCSs in this section.

In a MADM problem, there are the set of $m$ alternatives $R=\left\{R_{1}, R_{2}, \ldots, R_{m}\right\}$ and the set of $n$ attributes $B=\left\{B_{1}, B_{2}, \ldots, B_{n}\right\}$. Then, the weight of the attributes $\theta_{t}$ with $\theta_{t} \in[0,1]$ and $\sum_{t=1}^{n} \theta_{t}=1$ is considered. The evaluation information of each alternative on each attribute in the MADM problem can be represented by a NCN $r_{s t}=\left(<T_{s t}, U_{s t}, F_{s t}>,<t_{s t}, u_{s t}, f_{s t}>\right)(t=1,2, \ldots, n ; s=1,2, \ldots$, $m)$ with $T_{s t}, U_{s t}, F_{s t} \subseteq[0,1]$ and $t_{s t}, u_{s t}, f_{s t} \subseteq[0,1]$. So, the decision matrix with neutrosophic cubic information can be expressed as $R=\left(r_{s t}\right)_{m \times n}$. Thus the decision procedures are listed in the following:

Step 1: By considering the benefit and cost types of attributes, setup an ideal solution (ideal alternative) $r^{*}=\left\{r_{1}^{*}, r_{2}^{*}, \cdots, r_{n}^{*}\right\}$, where the desired NCNs $r_{t}^{*}(t=1,2, \ldots, n)$ are expressed by

$r_{t}^{*}=\left(\begin{array}{l}<\left[\max _{s}\left(T_{s t}^{-}\right), \max _{s}\left(T_{s t}^{+}\right)\right],\left[\min _{s}\left(U_{s t}^{-}\right), \min _{s}\left(U_{s t}^{+}\right)\right], \\ {\left[\min _{s}\left(F_{s t}^{-}\right), \min _{s}\left(F_{s t}^{+}\right)\right]>,<\max _{s}\left(t_{s t}\right), \min _{s}\left(u_{s t}\right), \min _{s}\left(f_{s t}\right)>}\end{array}\right)$ for the benefit attributes

or

$r_{t}^{*}=\left(\begin{array}{l}<\left[\min _{s}\left(T_{s t}^{-}\right), \min _{s}\left(T_{s t}^{+}\right)\right],\left[\max _{s}\left(U_{s t}^{-}\right), \max _{s}\left(U_{s t}^{+}\right)\right],\left[\max _{s}\left(F_{s t}^{-}\right), \max _{s}\left(F_{s t}^{+}\right)\right]>, \\ <\min _{s}\left(t_{s t}\right), \max _{s}\left(u_{s t}\right), \max _{s}\left(f_{s t}\right)>\end{array}\right)$ for the cost attributes.

Step 2: Compute the measure value between an alternative $R_{s}(s=1,2, \ldots, m)$ and the ideal solution $R^{*}$ by using Equation (4) or Equation (5) or Equation (6), and then obtain the values of $Z_{\theta 1}\left(R_{S}, R^{*}\right)$ or $Z_{\theta 2}\left(R_{s}, R^{*}\right)$ or $Z_{\theta 3}\left(R_{s}, R^{*}\right)(s=1,2, \ldots, m)$.

Step 3: Corresponding to the measure values of $Z_{\theta 1}\left(R_{s}, R^{*}\right)$ or $Z_{\theta 2}\left(R_{s}, R^{*}\right)$ or $Z_{\theta 3}\left(R_{s}, R^{*}\right)$, rank the alternatives in descending order and choose the best one regarding the bigger measure value.

Step 4: End.

\section{Decision-Making Example}

Two practical decision-making examples in real environments are given in this section to illustrate the applications of the developed MADM method in an NCS setting.

\subsection{Practical Example 1}

We consider the practical decision-making example adapted from Reference [30] for convenient comparison. Suppose that a sum of money is invested by an investment company for one of four potential alternatives: $R_{1}$ (a food company), $R_{2}$ (a transportation company), $R_{3}$ (a software company), and $R_{4}$ (a manufacturing company). Then the four alternatives are evaluated over the set of the three attributes: $H_{1}$ (the potential risk as the benefit type), $H_{2}$ (the growth as the benefit type), and $H_{3}$ (the environmental impact as the cost type). Then the importance of the three attributes is indicated by the weight vector $\theta=(0.32,0.38,0.3)$. The evaluation values of the four alternatives over the three attributes are given by NCSs $r_{s t}=\left(<T_{s t}, U_{s t}, F_{s t}>,<t_{s t}, u_{s t}, f_{s t}>\right)(t=1,2,3 ; s=1,2,3,4)$. Thus, the neutrosophic cubic decision matrix can be constructed as follows:

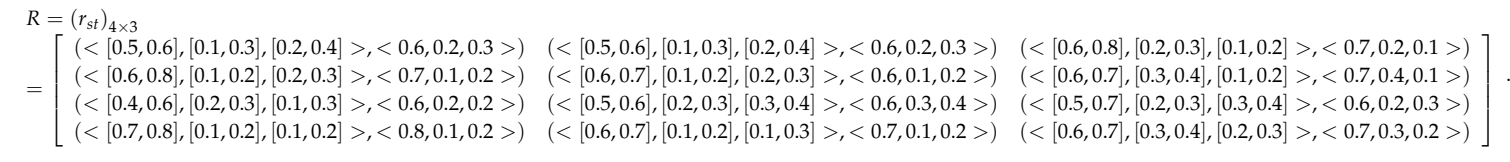

By the following steps, we use the proposed MADM method to judge which one is the best investment under an NCS environment.

First, when the ideal NCNs $r_{t}^{*}(t=1,2,3)$ of three attributes $H_{1}, H_{2}, H_{3}$ are obtained by 


$$
r_{t}^{*}=\left(\begin{array}{l}
<\left[\max _{s}\left(T_{s t}^{-}\right), \max _{s}\left(T_{s t}^{+}\right)\right],\left[\min _{s}\left(U_{s t}^{-}\right), \min _{s}\left(U_{s t}^{+}\right)\right],\left[\min _{s}\left(F_{s t}^{-}\right), \min _{s}\left(F_{s t}^{+}\right)\right]>, \\
<\max _{s}\left(t_{s t}\right), \min _{s}\left(u_{s t}\right), \min _{s}\left(f_{s t}\right)>
\end{array}\right) \text { for the benefit }
$$
attributes $H_{1}, H_{2}$

or

$$
\begin{aligned}
r_{t}^{*} & \text { or }
\end{aligned}\left(\begin{array}{l}
<\left[\min _{s}\left(T_{s t}^{-}\right), \min _{s}\left(T_{s t}^{+}\right)\right],\left[\max _{s}\left(U_{s t}^{-}\right), \max _{s}\left(U_{s t}^{+}\right)\right],\left[\max _{s}\left(F_{s t}^{-}\right), \max _{s}\left(F_{s t}^{+}\right)\right]>, \\
<\min _{s}\left(t_{s t}\right), \max _{s}\left(u_{s t}\right), \max _{s}\left(f_{s t}\right)>
\end{array}\right) \text { for the cost }
$$

attribute $\mathrm{H}_{3}$.

We can obtain an ideal solution (an ideal alternative) as follows:

$$
R^{*}=\left\{r_{1}^{*}, r_{2}^{*}, r_{3}^{*}\right\}=\left\{\begin{array}{c}
(<[0.7,0.8],[0.1,0.2],[0.1,0.2]>,<0.8,0.1,0.2>) \\
(<[0.6,0.7],[0.1,0.2],[0.1,0.3]>,<0.7,0.1,0.2>) \\
(<[0.5,0.7],[0.3,0.4],[0.3,0.4]>,<0.6,0.4,0.3>)
\end{array}\right\} .
$$

Second, by Equation (4) or Equation (5) or Equation (6), we compute the measure value between an alternative $R_{s}(s=1,2,3,4)$ and the ideal solution $R^{*}$. Then the measure values of $Z_{\theta 1}\left(R_{s}, R^{*}\right)$ or $Z_{\theta 2}\left(R_{s}, R^{*}\right)$ or $Z_{\theta 3}\left(R_{s}, R^{*}\right)(s=1,2,3,4)$ and the ranking of the alternatives are indicated in Table 1 .

Table 1. Measure results between the two NCSs $R_{S}$ and $R^{*}$ and ranking.

\begin{tabular}{cccc}
\hline$Z_{\theta m}\left(\boldsymbol{R}_{\boldsymbol{s}}, \boldsymbol{R}^{*}\right)$ & Measure Result & Ranking & The Best One \\
\hline$Z_{\theta 1}\left(R_{s}, R^{*}\right)$ & $0.9517,0.9822,0.9498,0.9945$ & $Z_{4}>Z_{2}>Z_{1}>Z_{3}$ & $Z_{4}$ \\
$Z_{\theta 2}\left(R_{s}, R^{*}\right)$ & $0.8246,0.9248,0.8474,0.9668$ & $Z_{4}>Z_{2}>Z_{3}>Z_{1}$ & $Z_{4}$ \\
$Z_{\theta 3}\left(R_{s}, R^{*}\right)$ & $0.9085,0.9654,0.9054,0.9893$ & $Z_{4}>Z_{2}>Z_{1}>Z_{3}$ & $Z_{4}$ \\
\hline
\end{tabular}

According to the results of Table 1 , the two alternatives $Z_{4}$ and $Z_{2}$ have the same ranking orders in all the measures, and $Z_{4}$ is the best choice.

\subsection{Related Comparison}

For convenient comparison, we select the MADM method introduced in the literature [30] as the related comparison. Then, we can get the measure values between $R_{s}$ and $R^{*}$ by the cosine measure $S_{w s}\left(R_{s}, R^{*}\right)(s=1,2,3,4)$ in [30], the standard deviation (SD), and the best choice, which are given in Table 2. Obviously, the SD values of our measures are bigger than the SD values of existing cosine measures. Therefore, our measures not only have good discrimination, but also get the same as the best choice $\left(Z_{4}\right)$, while existing cosine measures [30] indicate the different best choices $\left(Z_{4}\right.$ or $\left.Z_{2}\right)$. Thus, our measures have better decision-making robustness and discrimination than existing cosine measures [30].

Table 2. Related comparison of our measure results with existing cosine measure results.

\begin{tabular}{ccccc}
\hline Measure & Measure Value & Ranking Order & SD & The Best One \\
\hline$Z_{\theta 1}\left(R_{s}, R^{*}\right)$ & $0.9945,0.9822,0.9517,0.9498$ & $Z_{4}>Z_{2}>Z_{1}>Z_{3}$ & 0.0193 & $Z_{4}$ \\
$Z_{\theta 2}\left(R_{s}, R^{*}\right)$ & $0.9668,0.9248,0.8474,0.8246$ & $Z_{4}>Z_{2}>Z_{3}>Z_{1}$ & 0.0574 & $Z_{4}$ \\
$Z_{\theta 3}\left(R_{s}, R^{*}\right)$ & $0.9085,0.9654,0.9054,0.9893$ & $Z_{4}>Z_{2}>Z_{1}>Z_{3}$ & 0.0362 & $Z_{4}$ \\
$S_{w 1}\left(R_{1}, R^{*}\right)[30]$ & $0.9451,0.9794,0.9524,0.9846$ & $Z_{4}>Z_{2}>Z_{3}>Z_{1}$ & 0.0169 & $Z_{4}$ \\
$S_{w 2}\left(R_{2}, R^{*}\right)[30]$ & $0.9700,0.9906,0.9732,0.9877$ & $Z_{2}>Z_{4}>Z_{3}>Z_{1}$ & 0.0089 & $Z_{2}$ \\
$S_{w 2}\left(R_{2}, R^{*}\right)[30]$ & $0.9867,0.9942,0.9877,0.9968$ & $Z_{4}>Z_{2}>Z_{3}>Z_{1}$ & 0.0043 & $Z_{4}$ \\
\hline
\end{tabular}

\subsection{Practical Example 2}

Further, we give a real case about a punching machine to clearly demonstrate the usefulness of the proposed measures. There are four alternatives (design schemes), $R_{1}, R_{2}, R_{3}$, and $R_{4}$ in Table 3 . Then the four alternatives are evaluated over the set of three attributes: $H_{1}$ (manufacturing cost), 
$\mathrm{H}_{2}$ (structure complexity), and $\mathrm{H}_{3}$ (reliability). Then, the importance of the three attributes is indicated by the weight vector $\theta=(0.36,0.3,0.34)$. By the suitable evaluation of the four alternatives over the three attributes regarding NCNs $r_{s t}=\left(<T_{s t}, U_{s t}, F_{s t}>,<t_{s t}, u_{s t}, f_{s t}>\right)(t=1,2,3 ; s=1,2,3,4)$, the neutrosophic cubic decision matrix which is adapted from the literature [23] can be constructed as follows:

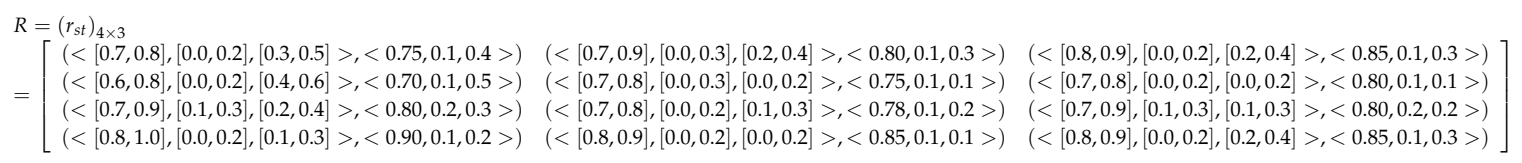

Table 3. Four alternatives (design schemes) of a punching machine [23].

\begin{tabular}{ccccc}
\hline Alternative & $\boldsymbol{R}_{\mathbf{1}}$ & $\boldsymbol{R}_{\mathbf{2}}$ & $\boldsymbol{R}_{\mathbf{3}}$ & $\boldsymbol{R}_{\mathbf{4}}$ \\
\hline Reducing mechanism & Gear reducer & Gear head motor & Gear reducer & Gear head motor \\
\hline Punching mechanism & Crank-slider mechanism & $\begin{array}{c}\text { Six bar } \\
\text { punching mechanism }\end{array}$ & $\begin{array}{c}\text { Six bar } \\
\text { punching mechanism }\end{array}$ & Crank-slider mechanism \\
\hline $\begin{array}{c}\text { Dial feed } \\
\text { intermittent mechanism }\end{array}$ & Sheave mechanism & Ratchet feed mechanism \\
\hline
\end{tabular}

By the following steps, we use the proposed MADM method to judge which one is the best design scheme under an NCS environment.

First, because we use a suitable evaluation of the four alternatives over the three attributes, all the benefit attributes are given in this decision problem. Thus, when the ideal NCNs $r_{t}^{*}(t=1,2,3)$ of the three attributes $H_{1}, H_{2}, H_{3}$ are obtained by $r_{t}^{*}=\left(\begin{array}{l}<\left[\max _{s}\left(T_{s t}^{-}\right), \max _{s}\left(T_{s t}^{+}\right)\right],\left[\min _{s}\left(U_{s t}^{-}\right), \min _{s}\left(U_{s t}^{+}\right)\right],\left[\min _{s}\left(F_{s t}^{-}\right), \min _{s}\left(F_{s t}^{+}\right)\right]>, \\ <\max _{s}\left(t_{s t}\right), \min _{s}\left(u_{s t}\right), \min _{s}\left(f_{s t}\right)>\end{array}\right)$, we can obtain an ideal solution (an ideal alternative) as follows:

$$
R^{*}=\left\{r_{1}^{*}, r_{2}^{*}, r_{3}^{*}\right\}=\left\{\begin{array}{c}
(<[0.8,1.0],[0.0,0.2],[0.1,0.3]>,<0.90,0.1,0.2>), \\
(<[0.8,0.9],[0.0,0.2],[0.0,0.2]>,<0.85,0.1,0.1>), \\
(<[0.8,0.9],[0.0,0.2],[0.0,0.2]>,<0.85,0.1,0.1>)
\end{array}\right\} .
$$

According to Equation (4) or Equation (5) or Equation (6), we can obtain the measure values of $Z_{\theta 1}\left(R_{s}, R^{*}\right)$ or $Z_{\theta 2}\left(R_{s}, R^{*}\right)$ or $Z_{\theta 3}\left(R_{s}, R^{*}\right)(s=1,2,3,4)$ and the ranking of all the alternatives, which are indicated in Table 4.

Table 4. Measure values between the two NCSs $R_{s}$ and $R^{*}$ and ranking.

\begin{tabular}{cccc}
\hline $\mathbf{Z}_{\theta \mathbf{m}}\left(\mathbf{R}_{\mathbf{s}}, \mathbf{R}^{*}\right)$ & Measure Value & Ranking & The Best One \\
\hline$Z_{\theta 1}\left(R_{s}, R^{*}\right)$ & $0.9683,0.9704,0.9847,0.9924$ & $Z_{4}>Z_{3}>Z_{2}>Z_{1}$ & $Z_{4}$ \\
$Z_{\theta 2}\left(R_{s}, R^{*}\right)$ & $0.8652,0.8937,0.8813,0.9701$ & $Z_{4}>Z_{2}>Z_{3}>Z_{1}$ & $Z_{4}$ \\
$Z_{\theta 3}\left(R_{s}, R^{*}\right)$ & $0.9386,0.9445,0.9699,0.9853$ & $Z_{4}>Z_{3}>Z_{2}>Z_{1}$ & $Z_{4}$ \\
\hline
\end{tabular}

According to the decision results in Table 4, they show that the two alternatives $Z_{4}$ and $Z_{1}$ have the same ranking orders in all the measures, with the best choice $Z_{4}$ and the worst choice $Z_{1}$.

If we set the same importance $\left(\theta_{t}=1 / 3\right.$ for $\left.t=1,2,3\right)$ of three attributes without considering the three attribute weights, we also obtained the same ranking with the attribute weights and without considering the three attribute weights in Table 5. It is obvious that the decision results of the proposed measures imply better robustness and lower sensitivity regarding attribute weights. 
Table 5. Measure values based on the different weights of the three attributes and ranking.

\begin{tabular}{|c|c|c|c|c|}
\hline$Z_{\theta m}\left(R_{s}, R^{*}\right)$ & $\begin{array}{c}\text { Measure Value } \\
\text { Based on } \theta=(0.36,0.3,0.34)\end{array}$ & $\begin{array}{c}\text { Measure Value } \\
\text { Based on } \theta=(1 / 3,1 / 3,1 / 3)\end{array}$ & Ranking & The Best One \\
\hline$Z_{\theta 1}\left(R_{s}, R^{*}\right)$ & $0.9683,0.9704,0.9847,0.9924$ & $0.9684,0.9697,0.9845,0.991$ & $Z_{4}>Z_{3}>Z_{2}>Z_{1}$ & $Z_{4}$ \\
\hline$Z_{\theta 2}\left(R_{S}, R^{*}\right)$ & $0.8652,0.8937,0.8813,0.9701$ & $0.8659,0.8927,0.8795,0.966$ & $Z_{4}>Z_{2}>Z_{3}>Z_{1}$ & $Z_{4}$ \\
\hline$Z_{\theta 3}\left(R_{s}, R^{*}\right)$ & $0.9386,0.9445,0.9699,0.9853$ & $0.9387,0.9432,0.9695,0.983$ & $Z_{4}>Z_{3}>Z_{2}>Z_{1}$ & $Z_{4}$ \\
\hline
\end{tabular}

\section{Conclusions}

This work proposed the Dice measure, cotangent measure, and Jaccard measure between two NCSs and discussed their properties. Then, we developed a MADM method based on one of three measures and applied it in real cases with neutrosophic cubic information. By comparison with an existing related MADM method, the proposed measures imply better robustness and lower sensitivity regarding attribute weights.

In this work, our main contributions are to enrich the neutrosophic cubic similarity measures and their decision-making method under NCS environments. In future work, the developed measures will be extended to medical/fault diagnosis and image processing.

Author Contributions: A.T. proposed the three measures of SNSs and their MADM method, and finished the initial draft; J.Y. provided the practical decision-making examples and related comparison; B.W. supervised the research activity planning and execution; then we wrote this paper together.

Funding: This research received no external funding.

Conflicts of Interest: The authors declare no conflict of interest.

\section{References}

1. Zadeh, L.A. Fuzzy sets. Inf. Control 1965, 8, 338-353. [CrossRef]

2. Smarandache, F. Neutrosophy: Neutrosophic Probability, Set, and Logic; American Research Press: Rehoboth, DE, USA, 1998.

3. Ye, J. A multicriteria decision-making method using aggregation operators for simplified neutrosophic sets. J. Intell. Fuzzy Syst. 2014, 26, 2459-2466.

4. Ye, J. Multicriteria decision-making method using the correlation coefficient under single-valued neutrosophic environment. Int. J. Gen. Syst. 2013, 42, 386-394. [CrossRef]

5. Liu, P.-D.; Wang, Y.-M. Multiple attribute decision-making method based on single valued neutrosophic normalized weighted Bonferroni mean. Neural Comput. Appl. 2014, 25, 2001-2010. [CrossRef]

6. Liu, P.D.; Wang, Y.M. Interval neutrosophic prioritized OWA operator and its application to multiple attribute decision making. J. Sci. Complex. 2016, 29, 681-697. [CrossRef]

7. Sahin, R. Cross-entropy measure on interval neutrosophic sets and its applications in multicriteria decision making. Neural Comput. Appl. 2017, 28, 1177-1187. [CrossRef]

8. Stanujkic, D.; Zavadskas, E.K.; Smarandache, F.; Brauers, W.K.M.; Karabasevic, D. A neutrosophic extension of the MULTIMOORA method. Informatica 2017, 28, 181-192. [CrossRef]

9. Ye, J. Improved cosine similarity measures of simplified neutrosophic sets for medical diagnoses. Artif. Intell. Med. 2015, 63, 171-179. [CrossRef] [PubMed]

10. Ye, J.; Fu, J. Multi-period medical diagnosis method using a single valued neutrosophic similarity measure based on tangent function. Comput. Methods Progr. Biomed. 2016, 123, 142-149. [CrossRef] [PubMed]

11. Cheng, H.D.; Guo, Y. A new neutrosophic approach to image thresholding. New Math. Nat. Comput. 2008, 4, 291-308. [CrossRef]

12. Guo, Y.; Sengur, A.; Ye, J. A novel image thresholding algorithm based on neutrosophic similarity score. Measurement 2014, 58, 175-186. [CrossRef]

13. Ye, J. Clustering methods using distance-based similarity measures of single-valued neutrosophic sets. J. Intell. Syst. 2014, 23, 379-389. [CrossRef]

14. Ye, J. Similarity measures between interval neutrosophic sets and their applications in multicriteria decision-making. J. Intell. Fuzzy Syst. 2014, 26, 165-172. 
15. Ye, J. Single valued neutrosophic cross-entropy for multicriteria decision making problems. Appl. Math. Model. 2014, 38, 1170-1175. [CrossRef]

16. Ye, J. Improved correlation coefficients of single valued neutrosophic sets and interval neutrosophic sets for multiple attribute decision making. J. Intell. Fuzzy Syst. 2014, 27, 2453-2462.

17. Zhang, H.-Y.; Ji, P.; Wang, J.-Q.; Chen, X.-H. An improved weighted correlation coefficient based on integrated weight for interval neutrosophic sets and its application in multi-criteria decision making problems. Int. J. Comput. Intell. Syst. 2015, 8, 1027-1043. [CrossRef]

18. Chen, J.; Ye, J. Some single-valued neutrosophic Dombi weighted aggregation operators for multiple attribute decision-making. Symmetry 2017, 9, 82. [CrossRef]

19. Zhang, H.-Y.; Wang, J.-Q.; Chen, X.-H. Interval neutrosophic sets and their application in multicriteria decision making problems. Sci. World J. 2014, 2014. [CrossRef] [PubMed]

20. Zhao, A.-W.; Du, J.-G.; Guan, H.-J. Interval valued neutrosophic sets and multi-attribute decision-making based on generalized weighted aggregation operator. J. Intell. Fuzzy Syst. 2015, 29, 2697-2706.

21. Sun, H.-X.; Yang, H.-X.; Wu, J.-Z.; Yao, O.-Y. Interval neutrosophic numbers Choquet integral operator for multi-criteria decision making. J. Intell. Fuzzy Syst. 2015, 28, 2443-2455. [CrossRef]

22. Biswas, P.; Pramanik, S.; Giri, B.C. TOPSIS method for multi-attribute group decision-making under single-valued neutrosophic environment. Neural Comput. Appl. 2016, 27, 727-737. [CrossRef]

23. Ye, J. Projection and bidirectional projection measures of single valued neutrosophic sets and their decision-making method for mechanical design schemes. J. Exp. Theor. Artif. Intell. 2017, 29, 731-740. [CrossRef]

24. Pouresmaeil, H.; Shivanian, E.; Khorram, E.; Fathabadi, H.S. An extended method using TOPSIS and VIKOR for multiple attribute decision making with multiple decision makers and single valued neutrosophic numbers. Adv. Appl. Stat. 2017, 50, 261-292. [CrossRef]

25. Jun, Y.B.; Kim, C.S.; Yang, K.O. Cubic sets. Ann. Fuzzy Math. Inform. 2012, 4, 83-98.

26. Jun, Y.B.; Smarandache, F.; Kim, C.S. Neutrosophic cubic sets. New Math. Nat. Comput. 2017, 13, 41-45. [CrossRef]

27. Ali, M.; Deli, I.; Smarandache, F. The theory of neutrosophic cubic sets and their applications in pattern recognition. J. Intell. Fuzzy Syst. 2016, 30, 1957-1963. [CrossRef]

28. Wang, H.; Smarandache, F.; Zhang, Y.-Q.; Sunderraman, R. Interval Neutrosophic Sets and Logic: Theory and Applications in Computing; Hexis: Phoenix, AZ, USA, 2005.

29. Wang, H.; Smarandache, F.; Zhang, Y.-Q.; Sunderraman, R. Single valued neutrosophic sets. Multispace Multistruct. 2010, 4, 410-413.

30. Lu, Z.K.; Ye, J. Cosine measures of neutrosophic cubic Sets for multiple attribute decision-making. Symmetry 2017, 9, 121. [CrossRef]

31. Banerjee, D.; Giri, B.C.; Pramanik, S.; Smarandache, F. GRA for multi attribute decision making in neutrosophic cubic set environment. Neutrosophic Sets Syst. 2017, 15, 60-69.

32. Pramanik, S.; Dalapati, S.; Alam, S.; Roy, T.K.; Smarandache, F. Neutrosophic cubic MCGDM method based on similarity measure. Neutrosophic Sets Syst. 2017, 16, 44-56.

33. Ye, J. Operations and aggregation method of neutrosophic cubic numbers for multiple attribute decision-making. Soft Comput. 2018. [CrossRef]

34. Shi, L.L.; Ye, J. Dombi aggregation operators of neutrosophic cubic sets for multiple attribute decision-making. Algorithms 2018, 11, 29. [CrossRef]

35. Ye, J. Vector similarity measures of simplified neutrosophic sets and their application in multicriteria decision making. Int. J. Fuzzy Syst. 2014, 16, 204-211.

36. Ye, J. The generalized Dice measures for multiple attribute decision making under simplified neutrosophic environments. J. Intell. Fuzzy Syst. 2016, 31, 663-671. [CrossRef]

37. Ye, J. Single valued neutrosophic similarity measures based on cotangent function and their application in the fault diagnosis of steam turbine. Soft Comput. 2017, 21, 817-825. [CrossRef]

(C) 2018 by the authors. Licensee MDPI, Basel, Switzerland. This article is an open access article distributed under the terms and conditions of the Creative Commons Attribution (CC BY) license (http:/ / creativecommons.org/licenses/by/4.0/). 